\title{
Sport e letteratura: Barthes, Delbée e Toussaint
}

\section{Margareth Amatulli}

\section{(2) OpenEdition}

\section{Journals}

\section{Edizione digitale}

URL: http://journals.openedition.org/studifrancesi/6170

DOI: 10.4000/studifrancesi.6170

ISSN: 2421-5856

\section{Editore}

Rosenberg \& Sellier

\section{Edizione cartacea}

Data di pubblicazione: 1 novembre 2010

Paginazione: 515-522

ISSN: 0039-2944

\section{Notizia bibliografica digitale}

Margareth Amatulli, «Sport e letteratura: Barthes, Delbée e Toussaint», Studi Francesi [Online], 162 (LIV | III) | 2010, online dal 30 novembre 2015, consultato il 08 janvier 2021. URL: http:// journals.openedition.org/studifrancesi/6170 ; DOI: https://doi.org/10.4000/studifrancesi.6170

\section{(c) $(1)(9)$}

Studi Francesi è distribuita con Licenza Creative Commons Attribuzione - Non commerciale - Non opere derivate 4.0 Internazionale. 


\section{Sport e letteratura: \\ Barthes, Delbée e Toussaint}

Che la pratica sportiva sia un vero e proprio testo da decodificare lo dimostra tutta una produzione letteraria che si avvale della mediazione del racconto per salvaguardare un'esperienza generalmente effimera, da cui trae origine e di cui si fa eco nel tempo. Dai primi 'resoconti' sportivi che figurano già nell'Iliade, in quelle cerimonie funebri, ad esempio, che sotto forma di gioco Achille organizza in onore dell'amico Patroclo, a Les Olympiques di Montherlant o a W ou le souvenir d'enfance di Georges Perec, lo sport fa la sua comparsa in modo diretto, metaforico, poetico o prosaico nella letteratura. La coesistenza delle due pratiche, sportiva e letteraria, ha indotto teorici e critici a leggere le diverse modalità attraverso cui il discorso letterario racconta in modo più o meno diretto lo sport, e a interrogarsi sulla natura e legittimità di una letteratura che lo elegge a tema. Una letteratura che, tramite la poesia, la finzione, l'autobiografia, la testimonianza, il saggio, mettendo in scena, alludendo o riflettendo su avvenimenti a essa generalmente estranei, si interroga tacitamente sul proprio grado di rappresentatività. Se il discorso letterario, infatti, si presta ad analizzare il fenomeno sportivo, reciprocamente lo sport pone alla letteratura la questione della sua identità.

La curiosità per questo mito collettivo dei tempi moderni ritorna più volte negli interessi di Roland Barthes; non solo nelle sue Mythologies del 1957 ma anche in un testo molto meno noto, Le sport et les hommes ${ }^{1}$, edito solo qualche anno fa e scaturito, negli anni '60, da un progetto di collaborazione con il romanziere, giornalista, produttore e regista quebecchese Hubert Aquin alle prese con un documentario sullo sport.

Cher Monsieur Barthes,

Je dois commencer sous peu la réalisation d'un film documentaire d'une heure sur le sport. Mon intention n'est pas de faire l'histoire du sport, mais plutôt, disons, sa phénoménologie et sa poétique.

Vous avez déjà deviné que votre premier chapitre de «MYTHOLOGIES» m'a beaucoup intéressé et que je viens, par cette lettre, vous demander d'écrire le commentaire de mon film. Mais il y a plus qu'une coïncidence car j'ai lu plusieurs de vos écrits et je me sens en accord avec votre vision du réel; et j'entends par ce «réel», aussi bien le Catch, Racine, que le Tour de France... ${ }^{2}$

Tale richiesta epistolare inoltrata il 4 aprile 1960 (e riprodotta in margine al libro) sanciva l'inizio di una fruttuosa collaborazione da cui è scaturito il film Le sport et les hommes trasmesso il $1^{\circ}$ giugno 1961 da Radio Canada. Il testo di Barthes,

(1) Roland Barthes, Le sport et les hommes. Texte du film "Le sport et les hommes" d'Hubert Aquin, Montréal, Les Presses de l'Université de Montréal,
2004, pp. 80.

(2) Ibid., p. 73 
dall'omonimo titolo, che, come fa notare Gilles Dupuis nella prefazione, non compare nell'edizione del 1995, né in quella ampliata del 2002 delle Euvres complètes edite da Seuil, viene pubblicato solo nel 2004 da Les Presses de l'Université de Montréal, e nel 2007 presentato nella traduzione italiana dalla casa editrice Einaudi ${ }^{3}$.

Lo sport e gli uomini si presenta come un'elegante plaquette in bianco e nero illustrata da alcune fotografie d'archivio (che sostituiscono, per questioni di diritti d'autore, le immagini originali di archivio e cronaca sportiva, provenienti da agenzie di tutto il mondo, con cui Aquin aveva confezionato il film), unitamente ai commenti, alle annotazioni e agli interrogativi del semiologo, estremamente funzionali alle sequenze cinematografiche. A strutturare il volume è la riflessione su cinque sport nazionali: la corrida in Spagna, la corsa automobilistica in Italia, il Tour de France ciclistico, l'hockey in Canada e il football in Ungheria, secondo le intenzioni del regista in parte condizionate dalla formula prestabilita della serie Paragoni cui è destinato il documentario.

I commenti di Barthes rispondono pienamente allo scopo di Hubert Aquin «de comprendre le sport, d'appréhender sous un éclairage rationnel nouveau ce phénomène social et poétique qui opère sur les foules une véritable cristallisation amoureuse» ${ }^{4}$. E infatti Barthes evidenzia nei suoi commenti la fenomenologia e la poetica degli sport esaminati che, al di là delle specifiche differenze, realizzano una comune lotta contro «la résistance des choses» ${ }^{5}$ e la loro «pesanteur obstinée» ${ }^{6}$. Al di là della «matière même d'une nation, c'est-à-dire de son sol et de son climat» ${ }^{7}$, che conferisce carattere nazionale agli sport, questi ultimi assolvono alla stessa funzione sociale, quella detenuta un tempo dal teatro: riunire la cittadinanza nell'esperienza comune, la conoscenza delle proprie passioni. Essi mettono alla prova identici valori morali: «endurance, sang-froid, témérité, courage»'; coinvolgono giocatori e spettatori in un'unica esperienza, perché «regarder, ici, c'est non seulement vivre, souffrir, espérer, comprendre, mais aussi et surtout le dire, de la voix, du geste, du visage, c'est prendre à témoin le monde entier: en un mot, c'est communiquer»?

Una concezione umanista dello sport trionfa sulla spettacolarizzazione della macchina e della forza fisica:

Non, le muscle ne fait pas le sport: voilà ce que dit le Tour de France. Le muscle, si précieux soit-il, n'est jamais qu'une matière première: ce n'est pas lui qui gagne.

Ce qui gagne, c'est une certaine idée de l'homme et du monde, de l'homme dans le monde ${ }^{10}$.

Ed è sempre l'essere umano a dover sconfiggere l'ignoranza, la paura, la necessità, come nella corrida, o a dominare il tempo, come nelle gare automobilistiche o ciclistiche. Ecco perché «la mort d'un coureur est [...] infiniment triste: car ce n'est pas seulement un homme qui meurt, c'est un peu de perfection qui disparaît de ce monde» ${ }^{11}$. Una perfezione mortale proprio perché umana. Inseguendo un'impossibile risposta al persistente interrogativo «Qu'est-ce que le sport?» che aleggia come un ritornello su tutto il testo, Roland Barthes fornisce un'acuta testimonianza sull'universo umano che stipula il suo contratto proprio attraverso la pratica sportiva, la cui essenza risiede interamente nella lotta.

(3) Lo sport e gli uomini, Torino, Einaudi, 2007,

pp. 62; trad. di Chiara BongIOvanNI.

(4) Roland BarTHEs, Le sport et les hommes cit., p. 74 .

(5) Ibid., p. 45.

(6) Ibid., p. 33.
(7) Ibid., p. 53.

(8) Ibid., p. 57.

(9) Ibid., pp. 65-67.

(10) Ibid., p. 51.

(11) Ibid., p. 27. 
Se «ce combat est une compétition [...] pas un conflit» ${ }^{12}$, secondo le parole del semiologo, cosa succede quando il mondo delle passioni altera impropriamente tale nobile essenza?

Il se produit alors une sorte de preuve du sport par l'absurde: c'est le scandale sportif.

Ce scandale a lieu lorsque les hommes rompent la mince barrière qui sépare les deux combats: celui du sport et celui de la vie. Ayant perdu tout intermédiaire, privé d'enjeu et de règle, le combat des joueurs cesse d'être soumis à la distance sans laquelle il n'y a pas de société humaine: il redevient un conflit.

Le sport retourne alors au monde immédiat des passions et des agressions, et il y entraîne la foule qui venait précisément lui demander de l'en purifier ${ }^{13}$.

\section{L'affaire Zidane}

La realtà sportiva degli ultimi tempi ha fatto più volte impropriamente irruzione nel mondo non mediato delle passioni e delle aggressioni di cui parla Barthes. Ad esempio, la testata di Zidane in risposta alle provocazioni verbali di Materazzi durante la finale della Coppa del Mondo 2006 non ha soltanto riempito fittamente le cronache mondiali, non è stata solo oggetto di ampie discussioni, di mille congetture, di interpretazioni sociologiche atte a rivendicare, ad esempio, il codice d'onore marsigliese dietro il gesto inaudito, ma ha ispirato anche la letteratura. Due libri in particolare hanno sfidato il grado di rappresentatività della letteratura trasformando un personaggio mediatico in un personaggio letterario. In $\mathrm{La} 107^{\circ}$ minute $^{14}$ e in $\mathrm{La}$ mélancolie de Zidane ${ }^{15}$ Anne Delbée e Jean-Philippe Toussaint interpretano, secondo i propri canoni espressivi e le rispettive preoccupazioni letterarie, l'avvenimento che ha fatto scalpore nello stadio di Berlino. Al di là delle naturali differenze, entrambi i testi si pongono come un calligrafico gesto di riabilitazione nei confronti dell'eroe calcistico che, ora sotto l'influenza di Apollo, ora sotto quella di Saturno, scelto dalla tragedia secondo Delbée, o raggiunto dalle divinità ostili della malinconia secondo Toussaint, è destinato alla solitudine e all'incomprensione.

Generalmente indifferente alle imprese del calcio, Anne Delbée, commediografa, specialista di Camille Claudel e Racine, autrice di libri sulla danza, la sera della finale Italia-Francia camminava per le vie deserte di Parigi assaporando l'aria dolce della sera che non lasciava presagire l'imminente tragedia. Perché, secondo Delbée, proprio di tragedia si tratta: in quel $107^{\circ}$ minuto dell'incontro sportivo sul quale di ritorno a casa accende la televisione, la scrittrice comprende che qualcosa di terribile sta avvenendo: trasformato in teatro antico, lo stadio diventa la scena in cui Zidane, eroe della tragedia greca, alla vittoria preferisce, a discapito dell'onore, una morte simbolica che gli assicurerà l'eternità.

Con uno sguardo di tenera comprensione, Delbée avverte l'urgenza di riscrivere la partita: «il fallait donc refaire le match, l'écrire dans un autre style. Il était temps de graver la tragédie au fronton du Théâtre» ${ }^{16}$; perché quella sera per l'autrice di teatro a essere in ballo non sono tanto il successo, il denaro facile, quanto «la renaissance de la tragédie dans toute la pureté de sa lumière. L'esprit flamboyant des mots dans le corps défait» ${ }^{17}$.

(12) Ibid., p. 45.

(13) Ibid., p. 61.

(14) Anne Delbée, La $107^{e}$ minute, Paris, Les Quatre Chemins, 2006, pp. 109.

(15) Jean-Philippe Toussaint, La mélancolie de Zidane, Paris, Minuit, 2006, pp. 19 (La melanconia di Zidane, Bellinzona, Casagrande, 2007, pp. 28; trad. it. di Roberto FERRUCCIO, con testo originale a fronte e una nota di Laurent Demoulin).

(16) AnNe DelbÉE, op. cit., p. 61.

(17) Ibid., p. 76. 
Ce soir-là, le sang n'avait pas encore coulé, et les spectateurs craignaient une finale trop prévisible avec couronnement et béatification de Zizou, lorsque Apollon, l'implacable, pénétra dans le stade, dressé sur son char. La divinité s'assura que le jeu touchait presque à sa fin avant de lancer son chant le plus pur.

Le Jeu n'aurait d'intérêt que s'il soulevait les passions, s'il révélait une liberté.

Zinedine Zidane était celui qu'Apollon ce soir-là avait désigné pour être sacrifié. L'autre n'était qu'un outil au service du dieu ${ }^{18}$.

E come la Phèdre di Racine per Roland Barthes, la tragedia che ha luogo nell'Olympiastadion di Berlino è, per la commediografa, innanzitutto una tragedia della parola che il giocatore avrebbe potuto evitare solo mostrandosi sordo al mondo:

Parce que vous, vous croyez aux mots, ce qu'ils disent, ce qu'ils signifient, ce qu'ils engendrent, ce qu'ils défendent.

Les mots n'ont aucune importance? Allons donc. [...]. Un mot de trop et les voisins s'entretuent, les clans se dressent les uns contre les autres, l'élève poignarde le maitre, et les politiques perdent le cœur des citoyens parce qu'ils manipulent trop et trop souvent les mots ${ }^{19}$.

\section{E ancora:}

S'il croyait aux mots, il ne pourrait pas ne pas réagir. S'il ne disait rien, il acceptait de trahir sa vérité d'homme.

Il provoqua le Joueur dans ce qu'il avait de plus cher. Il choisit les mots qui salissent, ceux qui pervertissent, qui dégradent ${ }^{20}$,

perché «bien au-delà de la mort, certaines paroles résonnent encore, peuvent salir votre mémoire, vous déshonorer au plus profond de votre cœur» ${ }^{21}$.

In un testo che alterna la terza persona con il "vous" interlocutorio diretto al calciatore francese, e evita opportunamente di nominare "l'autre" (a volte incautamente identificato con "le Malin") per ritrovarne il nome solo nelle ultime pagine del libro, Anne Delbée recupera arditamente personaggi e citazioni della mitologia greca e della cronaca moderna che, secondo i suoi occhi, compaiono in filigrana dietro ogni gesto di Zidane. Il suo pallone, ad esempio, è paragonato all'astro tentatore di Icaro: «Le ballon tel un astre vous guidait pour ce dernier envol. Icare une nouvelle fois s'approchait de la lumière, jusqu'à accepter de se brûler les ailes» ${ }^{22}$; lo scontro violento tra il calciatore e Materazzi fa eco a quello di Achille e Ettore: «Il y a presque trois mille ans. Achille, le Grec, acharné à mettre à mort Hector, le Troyen, tous deux semblablement agrippés l'un à l'autre dans la grande plaine antique ${ }^{23}$; «Et c'est Hector qui, une fois de plus, meurt. Il renonce à être un demi-dieu et en même temps il accepte la loi des hommes, mais déchu il laisse une trace avant de disparaitre» ${ }^{24}$. Il cartellino rosso dell'arbitro è la manifestazione del fato: «L'oracle s'est prononcé. CEdipe chassé doit reprendre sa route» ${ }^{25}$; la sofferenza del giocatore francese isolato dal suo pubblico è quella di Antigone che «descend lentement, vivante, les marches de son tombeau» e che, unica ad aver compreso il gesto insensato, «à travers trois mille ans d'histoire [le] rejoint, petite fille qui [le] prend doucement par la main ${ }^{26}$. Bandito

(18) Ibid., pp. 66-67.

(19) Ibid., p. 41.

(20) Ibid., p. 68.

(21) Ibid., pp. 17-18.

(22) Ibid., p. 57.
(23) Ibid., p. 15.

(24) Ibid., p. 34.

(25) Ibid., p. 48.

(26) Ibid., p. 51. 
dalla comunità, Zidane «à l'image des héros de la Tragédie va s'exclure de la société des hommes» ${ }^{27}$ per seguire le orme di Charlie Chaplin che «dans l'indifférence prend le bateau de l'exil» ${ }^{28}$ o per sostituirsi alla tristezza solitaria di Buster Keaton, in un silenzio simile a «celui du danseur à l'ultime ballet: plus jamais il n'enfilera les chaussons surhumains, celui de Jacques Brel en sueur couvert d'un peignoir dont l'âme rauque éclate pour la dernière fois en mille notes vers l'invisible spectateur» ${ }^{29}$.

La sovrabbondanza, spesso gratuita, di riferimenti letterari attorno a cui il testo si costruisce, il lirismo, a volte esasperato, che caratterizza la scrittura si stemperano come per incanto nell'ultima pagina del volume. Consapevole che un appuntamento misterioso ha suggellato l'incontro tra il calciatore e una scrittrice sino a quel momento disinteressata al calcio («Peut-être un dieu nous avait donné rendez-vous, moi qui ne regardais jamais les matches, vous qui ne prononciez que peu de mots» $\left.{ }^{30}\right)$, Anne Delbée recupera più autenticamente l'avvenimento:

J'ai pensé: «Chacun doit affronter sa $107^{\circ}$ minute au cours de sa vie. Certains la redoutent, d'autres la fuient, d'autres encore l'attendent, mais tous devront la vivre à la seconde près».

Hector était mort mais Andromaque comme chaque soir lui préparait son bain et tout redevenait vivant ${ }^{31}$.

Se nessuno, dunque, può sfuggire alla tragedia, niente può essere uguale dopo di essa tranne il racconto alla cui vitalità è affidata la perdita dell'eroe, e nel cui spazio la tragedia della parola prende la propria rivincita sulla finitezza del suo destino trasformandosi in parola della tragedia.

Per Jean Philippe Toussaint, invece, il gesto di Zidane è una questione di forma e di malinconia, La mélancolie de Zidane, appunto, come recita il titolo del brevissimo testo edito nel 2006 da Minuit che non si lascia catalogare facilmente in un genere preciso. Né romanzo, né semplice commento, né pamphlet, né novella, in meno di venti pagine la scrittura di Toussaint trasforma un protagonista assoluto del campo sportivo in un personaggio del campo letterario; un personaggio che «incapable de marquer un but, marquera les esprits $»^{32}$. Per l'autore che si era già sperimentato col mondo sportivo e in particolare calcistico, non si tratta di commentare la vicenda dal punto di vista giornalistico ma di apporre all'avvenimento la sua firma di scrittore. Gli articoli redatti, come spettatore, per Libération e per un giornale tedesco sulla coppa del mondo disputatasi precedentemente in Corea e Giappone dovevano, in una prima intenzione, essere raccolti in un volume che si sarebbe chiuso proprio con il resoconto della finale del 2006. La straordinarietà dell'evento che vede protagonisti Zidane e Materazzi travalica, per lo scrittore, l'importanza dello scenario sportivo e il testo che ne deriva, «beaucoup plus fort que le reste» ${ }^{33}$, viene pubblicato isolatamente in accordo con l'editore. Non si tratta, infatti, di un resoconto giornalistico dell'avvenimento, né di un'apologia sciovinista del 'gesto inaudito', quanto di una professione di fede nel potere della letteratura attraverso una lettura densa e personale di quello che, guarda caso, Toussaint definisce un gesto calligrafico, per la subitaneità e la scioltezza che lo hanno caratterizzato. Un gesto che, innanzitutto, non riesce a dominare la forma, «la forme à l'état pur» ${ }^{34}$ che resiste impavidamente allorché il calciatore cerca

(27) Ibid., p. 34.

(28) Ibid., p. 55.

(29) Ibid.

(30) Ibid., pp. 96-97.

(31) Ibid., pp. 108-109.

(32) Jean-Philippe Toussaint, op. cit., p. 14.
(33) Cfr. Magdalena Hrozinková, Jean-Philippe Toussaint, entre la Chine dans «Fuir» et le foot dans «La Mélancolie de Zidane» consultabile sul sito: http://www.radio.cz/fr/aricle/84694.

(34) Jean-Philippe Toussaint, La mélancolie de Zidane cit., p. 8. 
di sfidarla con un rigore che, solo per un fugace momento, sembra rivaleggiare con il tiro leggendario di Geoff Hurst a Wembley del 1966. Rimbalzato sulla linea e giudicato gol, il più controverso della storia del calcio, il gol non gol o gol fantasma, come venne stimato, in quella occasione decise inaspettatamente la vittoria della nazionale inglese. Lo stesso colpo di testa, «de toute beauté» ${ }^{35}$, respinto da Buffon, restituisce a Zidane il senso della propria «impuissance irrémédiable» ${ }^{36}$. «[Le] dernier geste de pure forme» ${ }^{37}$ che avrebbe potuto segnare l'uscita di scena del calciatore si rivela, quindi, fallimentare, privo di energia, vigore e volontà, e naturale prosieguo di un cedimento fisico espresso inconsciamente, secondo Toussaint, dalla fascia di capitano che scivola di continuo giù nonostante i tentativi di farla stare al suo posto.

Se la forma resiste ai tentativi di dominio del calciatore, incurante della bellezza del tiro, la malinconia che anima l'uomo determina il gesto fatale, corto circuito di tendenze contraddittorie che faticano ad armonizzarsi: bellezza e bassezza, violenza e passione; un gesto che proprio della forma si prende gioco: «Le geste de Zidane ignore les catégories esthétiques du beau ou du sublime, il se situe au-delà des catégories morales du bien et du mal, sa valeur, sa force ne tiennent qu'à leur adéquation irréductible à l'instant précis où il est survenu» ${ }^{38}$. E in quell'istante preciso in cui si consuma il gesto avventato, ad esplodere è in realtà una lunga e segreta genesi animata da due altrettanto contraddittorie correnti sotterranee che non riescono a trovare un compromesso: l'impossibilità e al tempo stesso la volontà di suggellare la fine di una folgorante carriera calcistica. Alla «fuite finale devant l'accomplissement de l'œuvre» ${ }^{39}$, secondo le parole di Freud che Toussaint cita riportando in nota la fonte, si aggiunge una corrente saturnina affinché l'uscita dalle scene del calcio sia la più rapida possibile. Mi sembra che sia proprio qui la ricchezza di questo minuscolo libro. Le due correnti parallele e contraddittorie che, agli occhi di Toussaint, hanno scatenato il gesto impulsivo non sono forse le stesse che animano i personaggi dei libri di questo autore? «Je partis brusquement et sans prévenir personne» ${ }^{40}$ : è il protagonista di La salle de bain ${ }^{41}$ che parla e che Toussaint cita direttamente nel raccontare di Zidane; è lo stesso personaggio che rimanda a oltranza la decisione finale di uscire dalla vasca da bagno in cui ha preso dimora; lo stesso personaggio che impulsivamente colpirà con le freccette la fronte della compagna quando questa lo pone dinanzi alla sua impotenza. Gesti così impulsivi e inspiegabili compaiono in quasi tutti i romanzi di Toussaint; romanzi che, tra l'altro, evitano accuratamente di sciogliere i nodi narrativi da cui avevano preso forma, eludendo, con tutta la forza della scrittura, il processo di raggiungimento della fine, come per Zidane, e raccogliendosi proprio attorno a quella forza. Toussaint è consapevole della propria strategia narrativa che esplicita quando cita Freud (la «fuite finale devant l'accomplissement de l'auvre», per l'appunto) o Starobinski.

«La mélancolie de Zidane est ma mélancolie, je la sais, je l'ai nourrie et je l'éprouve» ${ }^{42}$ : afferma mimeticamente lo scrittore che parafrasa spesso e cita direttamente ${ }^{43}$ più di una volta l'autore di L'encre de la mélancolie (ad esempio, «Quelque chose en

(35) Ibid., p. 13.

(36) Ibid.

(37) Ibid.

(38) Ibid., p. 9.

(39) Ibid., p. 12

(40) Ibid., p. 11.

(41) Jean-Philippe Toussaint, La salle de bain, Paris, Minuit, 1985.

(42) Jean-Philippe Toussaint, La mélancolie de Zidane cit., p. 12.
(43) O ancora, utilizza, combinandole diversamente, le parole del critico come fossero le proprie. Ad esempio a pagina 12 del testo di Toussaint leggiamo: «le monde devient opaque, les membres sont lourds $[\ldots] »$, una frase in cui riecheggiano le parole di Starobinski: «le monde devient opaque, lourd et lent $[\ldots ..] \gg$ (JEAN STAROBINSKI, L'encre de la mélancolie, «La Nouvelle Revue Française», mars 1963 , n. 123, p. 422). 
nous se tourne contre nous» ${ }^{44}$ ), a cui sembrerebbe attingere anche la citazione di Bachelard che segnala opportunamente tratta da L'Eau et les Rêves ${ }^{45}$. Per Starobinski, non a caso, «le domaine de la mélancolie est toujours celui des contraires surprenants», è sintomo di una relazione «malheureuse avec l'espace», di un rapporto non armonico «du dedans et du dehors»; può essere, in particolare, «quelque chose en nous [qui] se tourne contre nous $»^{46}$. Parole che riecheggiano in filigrana, quando non compaiono direttamente, nel libretto di Toussaint di cui il saggio del critico francese sembra fungere da ipotesto ${ }^{47}$.

Parlandoci di Zidane, attraverso Jean Starobinski, Toussaint ci fornisce la chiave di lettura esemplare delle sue opere invitandoci a individuare proprio nella malinconia l'essenza dei suoi personaggi, sollecitandoci, cioè, a comprenderli forse più che a giudicarli. Anche perché, secondo l'interpretazione dell'autore, dell'aggressività di Zidane, e non a caso di quella dei protagonisti dei suoi romanzi, non rimane altro che la pulsione sotterranea che la nutre e supera la sua materializzazione, il gesto stesso. Un gesto che, come nel caso dello stadio di Berlino, solo la telecamera ha potuto individuare ma che è sfuggito all'occhio degli spettatori. Mettendo in causa lo statuto della realtà e della percezione sensibile dell'occhio umano, per Jean-Philippe Toussaint quel gesto non ha mai avuto luogo perché nessuno spettatore ha potuto vederlo. E se mai a Zidane fosse venuto in mente di dare una testata, questa non avrebbe mai raggiunto il destinatario

car, chaque fois que la tête de Zidane aurait parcouru la moitié du chemin qui la séparait du torse de l'adversaire, il lui en serait resté encore une autre moitié à parcourir, puis une autre moitié, puis une autre moitié encore, et ainsi de suite éternellement, de sorte que la tête de Zidane, progressant toujours vers sa cible mais ne l'atteignant jamais [...] ne pourra pas, jamais, c'est physiquement et mathématiquement impossible [...] entrer en contact avec le torse de l'adversaire $[\ldots]^{48}$.

L'avvenimento, quindi, che per alcuni è costato la vittoria della Francia alla finale dei mondiali, non si è verificato alla luce di quel paradosso di Zenone, trasformatosi nella penna dello scrittore in quello di Zidane, con cui nel 1985, in una bella recensione di La salle de bain apparsa su «Critique», Gil Delannoi ${ }^{49}$ aveva interpretato il movimento immobile attorno a cui si costruisce il primo romanzo dello scrittore. Un commento che Toussaint aveva letto e probabilmente apprezzato ma che, come aveva dichiarato in un'intervista ${ }^{50}$, riservava alla lettura del critico non avendo egli intenzionalmente inserito nel suo romanzo tale teorema. Lo recupera adesso a testimonianza di come la letteratura, nelle sue fonti primarie e secondarie, si irradia e si autogenera all'infinito. Dice bene Laurent Demoulin nella Nota al testo riportata nell'edizione italiana: queste pagine non sono solo ironia, dimostrazione di letteratura e omaggio serio, ma anche «una specie di appropriazione, un'estensione del dominio della lette-

(44) Jean-Philippe Toussaint, La mélancolie de Zidane cit., p. 12; la frase è ripresa da Jean STAROBINSKI, art. cit., p. 411.

(45) In effetti Starobinski fa riferimento a «l'étang stymphalisé» che per Bachelard corrisponde all'immaginazione melancolica riportando una citazione dell'autore di L'Eau et les Rêves da cui Toussaint potrebbe aver ripreso l'ultima frase: «L'eau mêlée de nuit est un remords ancien qui ne veut pas dormir»; cfr. JEAN STAROBINSKI, art. cit., p. 411 e Jean-Philippe Toussaint, La mélancolie dé Zidane cit., p. 14.

(46) Cfr. JeAn Starobinski, art. cit., p. 417; p. 418; p. 411.

(47) Al di là delle più o meno dichiarate citazioni del testo di Starobinski, quest'ultimo mi sembra di capitale importanza per l'interpretazione delle opere di Toussaint, in particolare per La salle de bain, cui fornisce diversi e nuovi spunti di lettura.

(48) Jean-Philippe Toussaint, La mélancolie de Zidane cit., pp. 17-18.

(49) GIL DelannoI, Cruel Zénon, «Critique», 1985 , n. 463 , pp. $1198-1200$.

(50) Cfr. l'intervista all'autore sul sito $w w w-f a-$ k.kw.uni-paderborn.de/.../toussaint-interview-2001. btm. 
ratura» ${ }^{51}$ e, aggiungerei, chiave ermeneutica dell'opera dell'autore. Ad avere l'ultima parola è quindi sempre la letteratura, e la sua capacità - per citare ancora Jean Starobinski - «[de] transformer l'impossibilité de vivre en possibilité de dire» ${ }^{52}$.

MARGARETH AMATULLI

(51) Laurent Demoulin, Nota in La melanconia di Zidane cit., p. 26.
(52) JeAn StAROBINSKI, art. cit., p. 422. 